Original Research Paper

\title{
Fractal Dimension for the Characterization of Asphalt Mixture Properties
}

\author{
Giovanni Leonardi \\ Department of Civil, Energy, Environmental and Materials Engineering (DICEAM), \\ University of Reggio Calabria, Reggio Calabria, Italy
}

Article history

Received: 26-03-2016

Revised: 24-06-2016

Accepted: 13-07-2016

Email: giovanni.leonardi@unirc.it

\begin{abstract}
In the design of asphalt mixtures for paved roads, the shape of solid elements has a great importance. In asphalt concrete, the shape of aggregate particles influences the resistance, durability, stiffness, fatigue response and the required binder content of the mixture. Fractal geometry is more suitable to describe the irregularity of the shape of aggregate particles. This paper describes the influence of fractal dimension on the proprieties of asphalt concrete. Following an analytical and an experimental study, it was possible to find a correlation between Hot Mix Asphalt (HMA) mechanical proprieties and the fractal characteristics of the aggregate mixtures. The proposed approach allows to determine the optimal fractal dimension in order to select an appropriate aggregate gradation for the specific use. This fractal approach could be employed for predicting the characteristics of asphalt concretes, given as input the fractal dimension of the aggregate mixtures of the concrete materials.
\end{abstract}

Keywords: Fractal Dimension, Mixture, Pavement, Marshal Stability

\section{Introduction}

In recent years, fractal geometry theory has found widespread applications in many disciplines including material science and civil engineering. Some studies applied the fractal geometry to characterize the microstructural complexity of different types of aggregates and mixtures. In particular, several authors applied fractal concepts to describe the self-similarity of soils and mineral aggregates (Bartoli et al., 1991) and their size distributions were characterized by fractal dimension. So, fractal geometry was used to model hydraulic proprieties of soil and cement by Giménez et al. (1997; Atzeni et al., 2010), to study the porosity by Atzeni et al. (2008; Huang et al., 1999; Perrier et al., 1996) and to model the surface characteristics of cement pastes and concretes by Kokkalis and Panagouli (1998; Winslow, 1985).

Lange et al. (1994) use the concept of fractal dimension to describe the structure of pores defined through image analysis.

The influence of the shape of aggregate elements on mechanical proprieties of HMA is well known (Shklarsky and Livneh, 1964; Huber and Heiman, 1987; Arasan et al., 2011; Buonsanti et al., 2012a; 2012b; 2013). Euclidian geometry is not adequate to described the shape irregularity of the aggregates (Arasan et al., 2011). Fractal geometry is more suitable to describe this irregularity of aggregate particles.

The gradation fractal dimensions of asphalt mixture was used to investigate the influence of different fractal dimensions on the mechanical proprieties of HMA and different results were obtained (Chen et al., 2005; Peng and Sun, 2007; Ruihua et al., 2008; Arasan et al., 2010; Leonardi, 2010; Wang, 2011; Dong et al., 2012).

In this study, the relationship between fractal dimension and mechanical proprieties of HMA has been investigated.

\section{Fractal Geometry}

The fractal geometry was established by Mandelbrot (1982; Mandelbrot and Blumen, 1989) to describe irregular geometrical shapes (coastlines, branches of trees, clouds, etc.). Mandelbrot coined the word fractal (derived from the Latin fractus meaning "broken" or "fractured") for these complex forms in order to express that their peculiarity is a non-integer dimension, which is fractal (Peitgen and Richter, 1986; Peitgen et al., 1988; 1992). The concept of fractal can be understood by the examples of Cantor set and Koch curve (Giménez et al., 1997). 


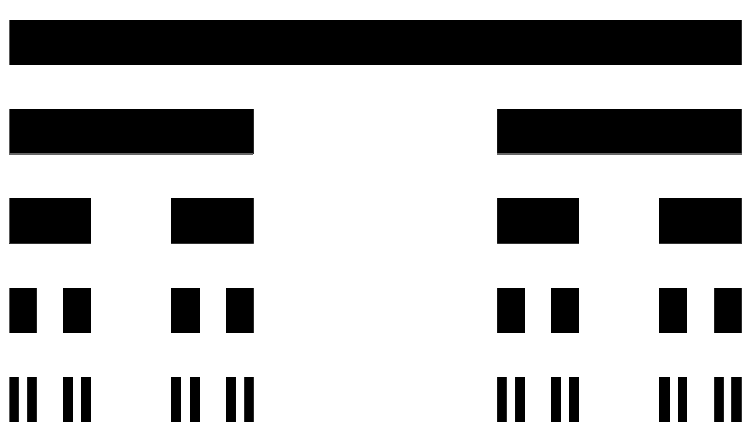

Fig. 1. Cantor bar

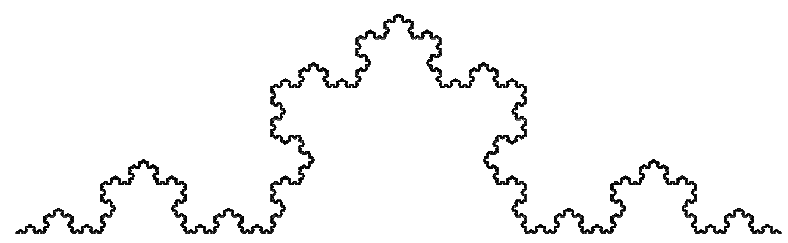

Fig. 2. Koch curve

The Cantor set (Fig. 1) is obtained by deleting recursively the middle third of a set of line segments (reductive algorithm). The Koch curve (Fig. 2) is also a solid line that is divided into three segments, but the middle segment is replaced with two lines equals in length of the deleted segment (accretive algorithm).

The above-illustrated fractals are characterized by their fractal dimensions. A fractal dimension is an index that measures the degree of complexity by evaluating how fast our measurements increase or decrease as our scale becomes larger or smaller (Paramanathan and Uthayakumar, 2008). Fractal dimension indicates the amount of space occupied, so the fractal dimension for a line varies between one and two and for a surface between two and three.

\section{Materials and Methods}

\section{Fractal Dimension of Aggregates Gradations}

Different fractal dimensions have been proposed for soil and aggregate applications. The mass fractal dimension, D, has been considered the most appropriate (Bartoli et al., 1991; Young and Crawford, 1991; Korvin, 1992). If the aggregate gradations are studied within the theory of fractal dimension, for particles of size $x$, the mass distribution function of aggregates could be written as:

$P(r)=\frac{M(r)}{M}=\frac{r^{3-D}-r_{\min }^{3-D}}{r_{\max }^{3-D}-r_{\min }^{3-D}}$

where, $M$ is the total mass of the aggregates; $M(r)$ is the mass of particles less than or equal in size to $r ; P(r)$ is the passing rate of aggregates; $r_{\max }$ and $r_{\min }$ are the maximum particle size and the minimum particle size. So different grading curves correspond to different values of $D$.

For fine aggregates, $r_{\min }$ is very small and can be neglected because of its irrelevant value; therefore Equation 1 can be transformed into:

$P(r)=\frac{M(r)}{M}=\left(\frac{r}{r_{\max }}\right)^{3-D}$

If the particle density $\rho$ is assumed to be constant, the volume is:

$V=\frac{M}{\rho}$

where, $M$ is the total mass of the aggregates and $\rho$ is the density of single particle.

Thus Equation 2 could be rewritten as Equation 4:

$P(r)=\frac{M(r)}{M}=\frac{V(r)}{V}=\left(\frac{r}{r_{\max }}\right)^{3-D}$

where, $V(r)$ is the fractal total volume of aggregates which particle sizes are less than $r$.

Consequently, the mass distribution of the fine aggregates is the same as the volume distribution and can both be described by using fractal dimension.

Hence:

$3-D=\frac{\ln P(r)}{\ln \left(\frac{r}{r_{\text {max }}}\right)}$

According to fractal dimension, the variation range of the fractal dimension of the aggregate the topological dimension and the dimension of the space, that is, $2<\mathrm{D}$ $<3$ (Dong et al., 2012).

This fractal representation could be extended to investigate the existing correlation between mechanical characteristics of the asphalt mixtures and fractal dimension.

The aggregate-size distribution is one of the main factors that influence the behavior of asphalt paving mixtures. In fact, the target density level of HMA is strongly related to aggregate gradation (Goode and Lufsey, 1962).

Goode and Lufsey (1962) demonstrated that an aggregate having a gradation that produces a straight line on a 0.45 in a power chart gradation will have the highest strength and the maximum achievable density and subsequently the minimum Voids in the Mineral Aggregate (VMA) in an HMA mixture. 
However, Huber and Shuler (1992) note that significant confusion exists concerning different methods used to draw aggregate gradation "maximum" density lines. Closely related to maximum density lines and also in debate, is the definition of nominal aggregate maximum size.

Table 1. Fractal dimensions of the nine aggregates mixtures

\begin{tabular}{llllllllll}
\hline Mixture & M1 & M2 & M3 & M4 & M5 & M6 & M7 & M8 & M9 \\
\hline Fractal dimension D & 2.513 & 2.552 & 2.394 & 2.256 & 2.394 & 2.463 & 2.478 & 2.483 & 2.455 \\
\hline
\end{tabular}

Table 2. Fractal dimensions $D$ and mechanical proprieties of the nine aggregates mixtures

\begin{tabular}{|c|c|c|c|c|c|c|c|c|c|}
\hline Mixture number & M1 & M2 & M3 & M4 & M5 & M6 & M7 & M8 & M9 \\
\hline Fractal dimension $D$ & 2.51 & 2.55 & 2.39 & 2.26 & 2.39 & 2.46 & 2.48 & 2.48 & 2.46 \\
\hline Stability $(k N)$ & 11.35 & 12.40 & 10.73 & 9.05 & 12.55 & 11.64 & 11.99 & 11.94 & 12.57 \\
\hline Marshall Quotient ( $\mathrm{kN} / \mathrm{mm})$ & 4.58 & 4.29 & 6.17 & 5.77 & 5.43 & 5.71 & 5.60 & 5.82 & 5.69 \\
\hline Porosity (\%) & 5.59 & 5.31 & 11.90 & 12.05 & 9.00 & 6.54 & 5.23 & 6.21 & 6.82 \\
\hline Density $\left(\mathrm{kg} / \mathrm{m}^{3}\right)$ & 2.40 & 2.41 & 2.24 & 2.22 & 2.31 & 2.34 & 2.36 & 2.34 & 2.33 \\
\hline
\end{tabular}

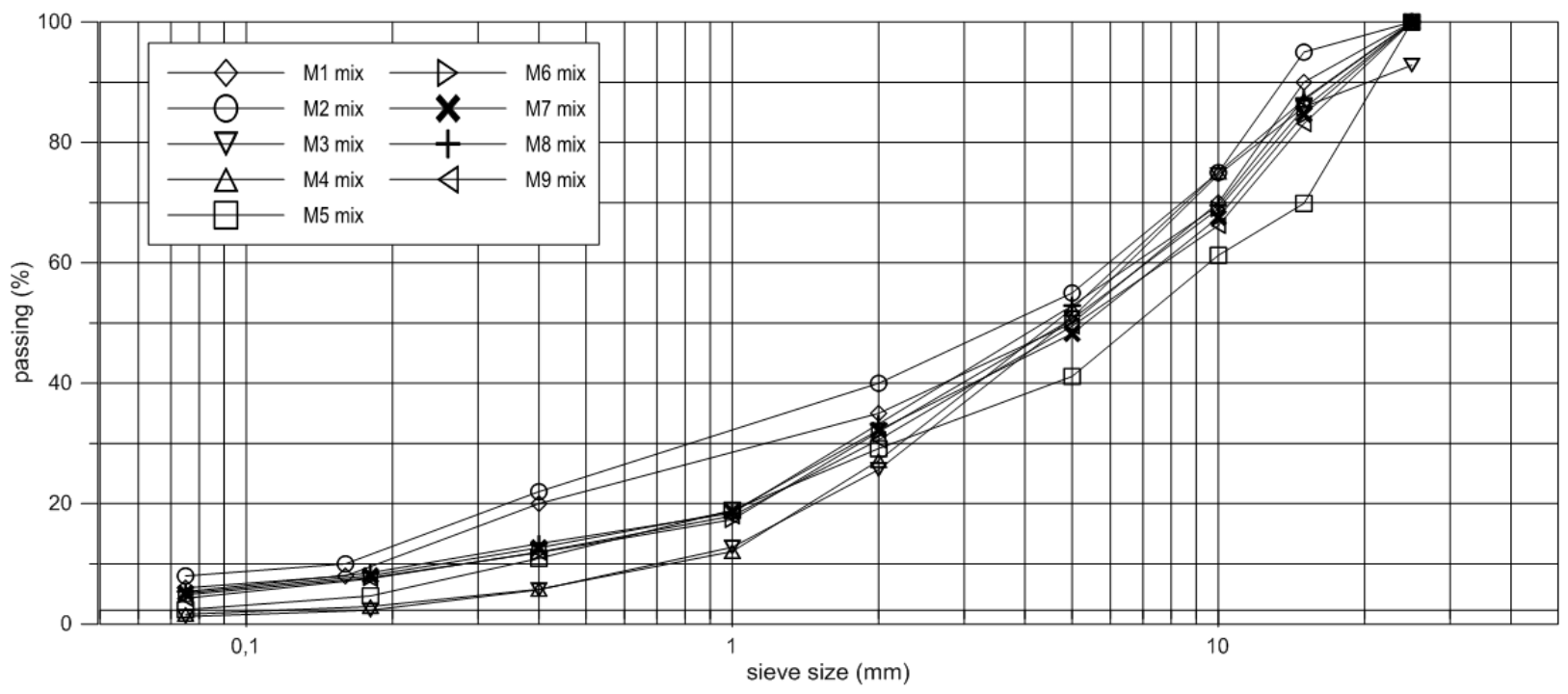

Fig. 3. Gradation of the considered nine aggregate mixtures

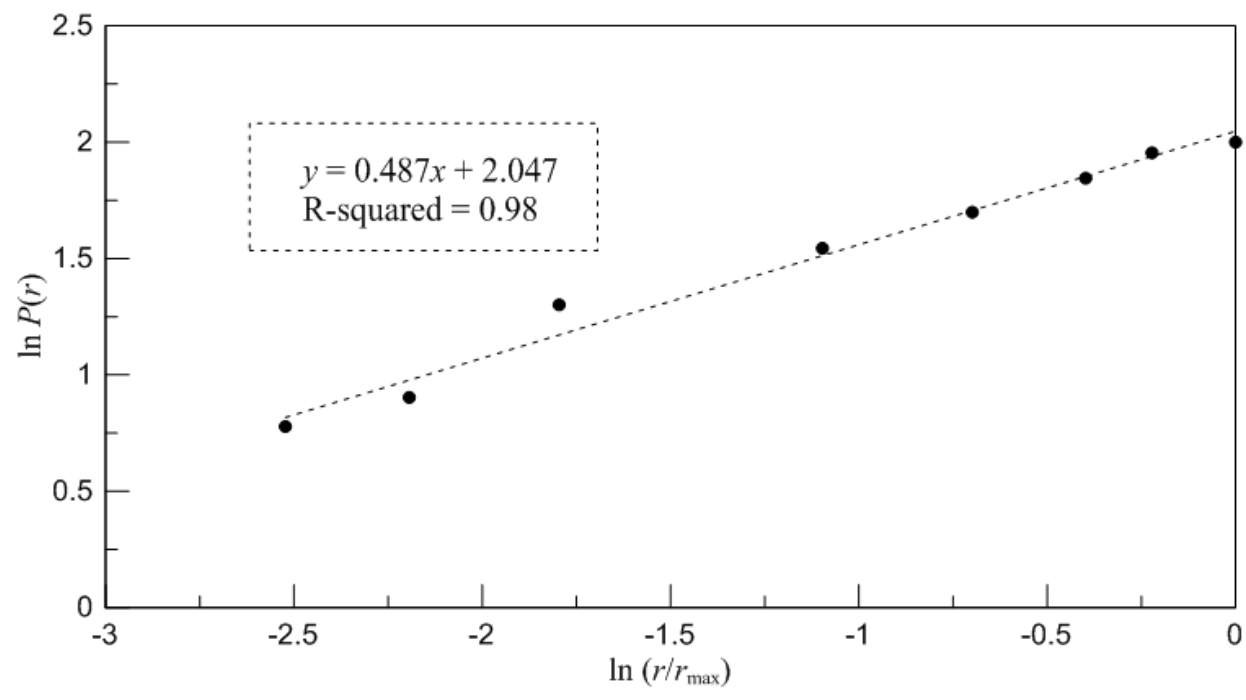

Fig. 4. Example of linear regression for the determination of the fractal dimension 
The purpose of this paper is to find a simple, accurate method to optimize the mixture design demonstrating that an aggregate mixture having a gradation that produces an optimal fractal dimension will have the maximum achievable density and subsequently the lowest voids in an HMA. To reach this aim, nine series of fine aggregate particle size distribution were designed. The nine aggregate gradation curves are shown in Fig. 3.

According to Equation 5 the gradation fractal dimension $D$ is relative to the slope of the function $\ln$ $P(r)-\ln \left(r / r_{\max }\right)$. In Fig. 4, the slope of the linear regression was 0.487 that corresponds, for the mixture number 1 , to a fractal dimension $3-0.487=2.513$.

The same method can be used to compute the fractal dimension of all the nine mixtures (Table 1).

The nine aggregate mixtures were used to form nine different Hot Mixed Asphalt concretes (HMA) in order to investigate the researched correlations. The asphalt mixes have the same type and quantity $(4.30 \%)$ of binder (asphalt).

\section{Results and Discussion}

The calculated fractal dimension of aggregates, the Marshall stability, the Marshall Quotient (MQ), the porosity and the density of the nine asphalt mixtures are reported in Table 2 .

As expected, it should be noted that the density values increase with the fractal dimension, while the porosity values decrease (Fig. 5).

The fractal dimension of the aggregates can also be linked to the mechanical proprieties of asphalt concrete. The obtained experimental results are shown in the following figures.

The correlation between fractal dimension and Marshall Stability and MQ of asphalt concrete are presented in Fig. $6 \mathrm{a}$ and $6 \mathrm{~b}$, respectively. It could be seen that the Marshall Stability increases with the increase in fractal dimension. This is an expected result because higher fractal dimension values represent higher aggregate surface irregularities and it is well known that increasing aggregates irregularities increases stability (Ishai and Gelber, 1982).

Figure $6 \mathrm{~b}$ shows the relationship between Marshall Quotients and fractal numbers. It is possible to observe the existence of an optimal value of the fractal dimension, this value can be estimated in 2.35. For greater values we can image an opposite trend of the variable. The MQ could be considered as an indicator of the resistance against the deformation of the asphalt mixture, a higher value of MQ could indicate a stiffer and more resistant mixture.

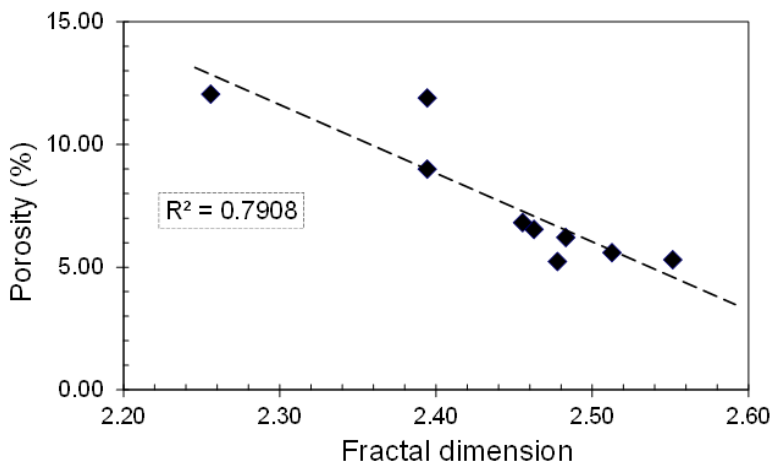

(b)

Fig. 5. Relationships between $D$ and density (a), $D$ and porosity (b)

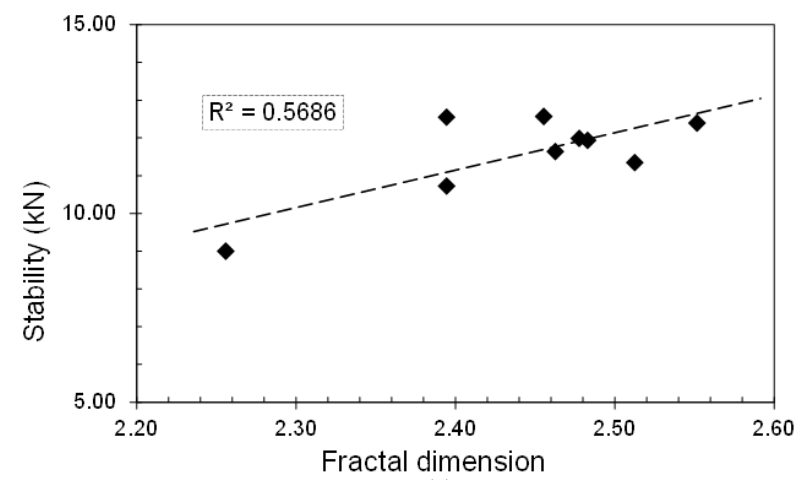

(a)

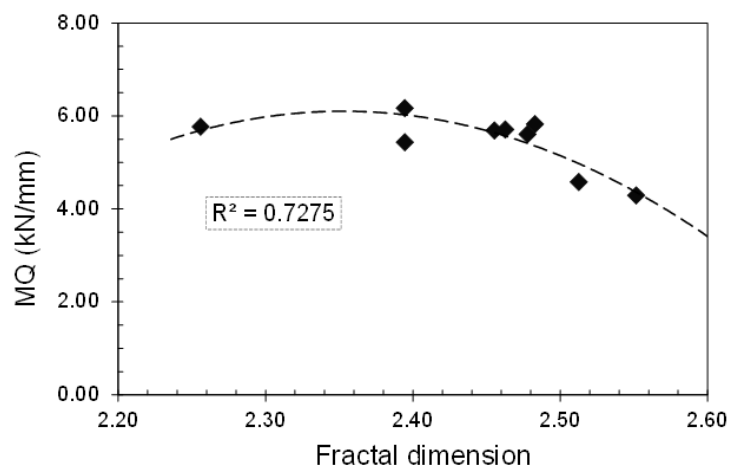

(b)

Fig. 6. Relationships between fractal dimension $D$ and Marshall Test results 
This result indicates the importance of fractal dimensions of aggregates for asphalt mixtures mechanical proprieties. Therefore, the fractal dimension of aggregate can be applied as the standard for the selection of aggregates.

\section{Conclusion}

The principal aim of the study was the application of fractal geometry for the characterization of the proprieties of asphalt concrete. The results confirm the good fit and reliability of the approach.

The fractal dimension represents an appropriate parameter in order to determine an adequate aggregate gradation that can guarantee the achievement of the required characteristics for the pavement. The illustrated results shows that there is a strong relationship between fractal dimension and the HMA characteristics.

Hence, it may be said, for example, that starting from the required mechanical properties of asphalt concrete, chosen for a particular type of pavement, it is possible to go back to the corresponding fractal dimension and consequently to design easily the aggregates mixture.

\section{Acknowledgment}

The author would like to gratefully acknowledge the unknown reviewers for their review and comments.

\section{Ethics}

This article is original and contains unpublished material.

\section{References}

Arasan, S., E. Yener, F. Hattatoglu, S. Akbulut and S. Hinislioglu, 2010. The relationship between the fractal dimension and mechanical properties of asphalt Concrete. Int. J. Civil Structural Eng., 1: 165-170.

Arasan, S., S. Akbulut and A.S. Hasiloglu, 2011. The relationship between the fractal dimension and shape properties of particles. KSCE J. Civil Eng., 15: 1219-1225. DOI: 10.1007/s12205-011-1310-X

Atzeni, C., G. Pia and U. Sanna, 2010. A geometrical fractal model for the porosity and permeability of hydraulic cement pastes. Constr. Build. Mater., 24: 1843-1847.

DOI: 10.1016/j.conbuildmat.2010.04.020

Atzeni, C., G. Pia, U. Sanna and N. Spanu, 2008. A fractal model of the porous microstructure of earth-based materials. Constr. Build. Mater., 22: 1607-1613.

DOI: $10.1016 /$ j.conbuildmat.2007.06.004
Bartoli, F., R. Philippy, M. Doirisse, S. Niquet and M. Dubuit, 1991. Structure and self-similarity in silty and sandy soils: The fractal approach. Eur. J. Soil Sci., 42: 167-185. DOI: $10.1111 / \mathrm{j} .1365-2389.1991 . t b 00399 . \mathrm{x}$

Buonsanti, M., G. Leonardi and F. Scopelliti, 2012a. Theoretical and computational analysis of airport flexible pavements reinforced with geogrids. RILEM Bookseries, 4: 1219-1227. DOI: 10.1007/978-94-007-4566-7_116

Buonsanti, M., G. Leonardi, F. Scoppelliti and F. Cirianni, 2012b. Dynamic behavior of granular mixture solids. Key Eng. Mater., 488-489: 541-544. DOI: $10.4028 /$ www.scientific.net/KEM.488-489.541

Buonsanti, M., G. Leonardi and F. Scoppelliti, 2013. Modelling micro-damage in granular solids. Key Eng. Mater., 525-526: 497-500. DOI: 10.4028/www.scientific.net/KEM.525-526.497

Chen, G., Y. Tan, Z. Wang and Y. Zhang, 2005. Fractal study of grading curve trend of the asphalt mixtures. J. Highway Transport. Res. Dev., 22: 1-4.

Dong, W., X. Guo and L. Gu, 2012. Study on the fractal gradation optimization of ATB-25. Proceedings of the 2nd International Conference on Electronic and Mechanical Engineering and Information Technology, (EIT' 12), Atlantis Press. DOI: 10.2991/emeit.2012.87

Giménez, D., E. Perfect, W.J. Rawls and Y. Pachepsky, 1997. Fractal models for predicting soil hydraulic properties: A review. Eng. Geol., 48: 161-183. DOI: 10.1016/s0013-7952(97)00038-0

Goode, J. and L. Lufsey, 1962. A new graphical chart for evaluating aggregate gradation.

Huang, S., Y. Yu, T. Lee and T. Lu, 1999. Correlations and characterization of porous solids by fractal dimension and porosity. Phys. A: Stat. Mech. Applic., 274: 419-432. DOI: 10.1016/S0378-4371(99)00299-X

Huber, G. and G. Heiman, 1987. Effect of asphalt concrete parameters on rutting performance: A field investigation (with discussion). Association of Asphalt Paving Technologist.

Huber, G. and T. Shuler, 1992. Providing sufficient void space for asphalt cement: Relationship of mineral aggregate voids and aggregate gradation. Effects of Aggregates and Mineral Fillers on Asphalt Mixture Performance.

Ishai, I. and H. Gelber, 1982. Effect of geometric irregularity of aggregates on the properties and behavior of bituminous concrete. Association of Asphalt Paving Technologists Proceedings.

Kokkalis, A. and O. Panagouli, 1998. Fractal evaluation of pavement skid resistance variations. I: Surface wetting. Chaos Solitons Fractals, 9: 1875-1890. DOI: 10.1016/S0960-0779(97)00138-0 
Korvin, G., 1992. Fractal Models in the Earth Sciences. 1st Edn., Elsevier Science Ltd., Amsterdam, ISBN-10: 0444889078, pp: 424.

Lange, D.A., H.M. Jennings and S.P. Shah, 1994. Image analysis techniques for characterization of pore structure of cement-based materials. Cement Concrete Res., 24: 841-853.

DOI: $10.1016 / 0008-8846(94) 90004-3$

Leonardi, G., 2010. Fractal dimension for the characterization of the porosity of asphalt concretes. Arch. Civil Eng., 56: 321-333.

DOI: $10.2478 / \mathrm{v} .10169-010-0018-5$

Mandelbrot, B. and A. Blumen, 1989. Fractal geometry: What is it and what does it do? [and Discussion]. Proc. Royal Society London, 423: 3-16. DOI: $10.1098 /$ rspa.1989.0038

Mandelbrot, B., 1982. The Fractal Geometry of Nature. 1st Edn., WH Freeman, ISBN-10: 0716711869, pp: 468.

Paramanathan, P. and R. Uthayakumar, 2008. Application of fractal theory in analysis of human electroencephalographic signals. Comput. Biol. Med., 38: 372-378. DOI: 10.1016/j.compbiomed.2007.12.004

Peitgen, H., D. Saupe and M. Barnsley, 1988. The Science of Fractal Images. Springer, New York, ISBN-10: 0387966080, pp: 312.

Peitgen, H.O. and P.H. Richter, 1986. The Beauty of Fractals. 1st Edn., Springer-Verlag New York, ISBN-10: 3540158510, pp: 199.
Peitgen, H.O., H. Jürgens and D. Saupe, 1992. Bausteine des Chaos Fraktale. 1st Edn., Springer Berlin Heidelberg, Berlin, ISBN-10: 3540557814, pp: 514.

Peng, Y. and L. Sun, 2007. Evaluating asphalt mixture homogeneity based on fractal theory. J. Harbin Inst. Technol., 10: 36-36.

Perrier, E., C. Mullon, M. Rieu and G. De Marsily, 1996. Computer construction of fractal soil structures: Simulation of their hydraulic and shrinkage properties. Water Resour. Res., 33: 259A-260A. DOI: 10.1029/95WR02214

Ruihua, Y., X. Zhihong, Z. Chao and L. Shuming, 2008. Fractal gradation theory of asphalt mixture. J. Tongji Univ. Nat. Sci., 12: 36-36.

Shklarsky, E. and M. Livneh, 1964. The use of gravels for bituminous paving mixtures. Proc. Assoc. Asphalt Pav. Technol., 33: 584-610.

Wang, L., 2011. Fractal dimension analysis of the fine aggregate gradation of interlocking skeleton asphalt mixture. J. Wuhan Univ. Technol.-Mater, 26: 567-572. DOI: 10.1007/s11595-011-0269-2

Winslow, D.N., 1985. The fractal nature of the surface of cement paste. Cement Concrete Res., 15: 817-824. DOI: $10.1016 / 0008-8846(85) 90148-6$

Young, I. and J. Crawford, 1991. The fractal structure of soil aggregates: Its measurement and interpretation. J. Soil Sci., 42: 187-192. DOI: $10.1111 / \mathrm{j} .1365-2389.1991 . t b 00400 . \mathrm{x}$ 